Victoriano Gaviño Rodríguez, Cádiz

\title{
Delimitación de conceptos lingüísticos. Definición y descripción de la finalidad
}

\begin{abstract}
This work represents an attempt to emphasize the problem that terminological or conceptual vagueness causes for linguistic studies, mainly in relation with the operations of definition and description. The distinction between definition and description is fundamental for linguists, but not everyone takes it into consideration. Descriptive grammar must not study the universal definition of concepts, but it needs to use them as previous base to identify correctly its extension and to describe the characteristics of the respective elements in several languages.

Based on the problem of a clear concept called 'purpose' in linguistic studies, we intend to suggest the following: on one hand, we intend to demonstrate a series of basic guidelines to study 'purpose' as linguistic universal; on the other hand, we try to apply them to the elements that belong to it in a specific language, in this case, spanish language.
\end{abstract}

Key words

Purpose, definition, description, concept, terminology

\section{Introducción}

Los estudios lingüísticos están plagados de términos especializados con los que frecuentemente aludimos a nociones lingüísticas ampliamente conocidas pero no por ello exentas de ambigüedades o vaguedades. Es precisamente el uso continuado que trabajo tras trabajo realizamos de términos como finalidad, causa, consecuencia, complemento indirecto, complemento circunstancial, colocación, traslación, etc., el que hace que en muy pocas ocasiones nos paremos a reflexionar si con el empleo de estos estamos aludiendo a verdaderos conceptos que den respuestas a los fenómenos que pretendemos explicar o si, por el contrario, con ellos incurrimos en contradicciones que ponen en marcha falsas inferencias que impiden analizar correctamente los elementos lingüísticos. Lamentablemente en no pocos casos la situación está más cercana a esta última perspectiva y el uso de muchos términos lingüísticos obedece más a una cuestión histórica que a una necesidad de los mismos para nuestras explicaciones, máxime cuando por medio de estos términos o no nos referimos a clases cerradas (con límites y caracterizaciones precisas) o no analizamos verdaderos fenómenos lingüísticos, sino hechos de la realidad que nos rodean disfrazados de una referencia pseudolingüística que se logra por medio del trazado de un puente que nos lleva de lo linguístico a lo extralingüístico. ${ }^{1}$ Sin embargo, seguimos obcecados en continuar el camino que nos dicta la tradición y nos obliga a lidiar una y otra vez con los mismos problemas de siempre, en lugar de abrir una brecha y batallar en contra de presupuestos defendidos por muchos autores durante años o siglos. Es cierto que en no pocas ocasiones la tradición nos presenta líneas seguras y caminos de solución a los fenómenos que abordamos, pero también, del mismo modo, es ella la que nos lega debilidades, enredos, problemas y en definitiva errores con los que tenemos que luchar día a día y de los que difícilmente podemos salir si, en

1 Muchos de los problemas interpretativos que encontramos en los estudios gramaticales actuales radican en la continua confusión entre lo lingüístico y lo extralingüístico, entre la lengua y sus referentes, que "ha metido los estudios lingüísticos en caminos de los que no es fácil salir, aunque no dan cuenta de manera satisfactoria del funcionamiento de la lengua" (cf. F. Matte Bon, 2007). Para un desarrollo de esta idea, cf. también lo defendido en F. Matte Bon (1999). 
lugar de soltarnos para encontrar un nuevo camino que recorrer, nos quedamos agarrados a las rocas como mejillones esperando a ser capturados. Si los términos o las explicaciones actuales no nos satisfacen o no resuelven los problemas lingüísticos con que nos encontramos, no nos queda más remedio que buscar nuevas caracterizaciones para estos términos o bien nuevos conceptos con los que poder abordar el estudio de los fenómenos linguiísticos.

\section{La finalidad en los estudios lingüísticos actuales}

El término 'finalidad', con el que trabajaremos en este artículo, no representa ninguna excepción en este punto. Si revisamos las distintas caracterizaciones que de la finalidad aportan los estudios linguiísticos, observaremos que las distintas perspectivas adoptadas hasta el momento nos conducen a un mar de indefiniciones al que tenemos que poner límite si no queremos adentrarnos en aguas más profundas y perdernos inevitablemente sin saber cómo salir de la corriente que nos lleve mar adentro. Bastaría una breve revisión historiográfica acerca de la noción de finalidad en los estudios gramaticales para concluir que, en líneas generales, esta ha sido considerada casi siempre un aspecto marginal, en los que generalmente nos topamos con unas mínimas líneas, sin duda insuficientes para la solución de las muchas dificultades que plantea su estudio. Y esto es así porque, para los estudios gramaticales, la finalidad nunca ha sido un objeto de estudio en sí misma: generalmente ha sido utilizada como mero instrumento en la caracterización de otras cuestiones consideradas más importantes, como son el estudio de los valores de los casos latinos, la caracterización del complemento indirecto o del complemento circunstancial, entre otros tantos. ${ }^{2}$ Por otro lado, cuando se ha abordado el tema -en la tradición hispánica, destacan, por ejemplo, los trabajos de C. Galán Rodríguez (1992), (1993) y (1999), S. García (1996) y A. Narbona Jiménez (1985)-, la situación no es tampoco más complaciente, pues los apuntes de los autores se limitan a la caracterización de las expresiones finales en una lengua concreta, sin que previamente se lleve a cabo una previa delimitación de la finalidad como noción gramatical (pues para todos es presupuesta), situación que acaba provocando su indeterminación y la aparición de continuos desórdenes entre su definición y la descripción de las características de los objetos que forman parte del concepto en cada lengua determinada, en nuestro caso, en español. Esta distinción entre definición y descripción, que nos parece fundamental para la labor del lingüista, no se ha llevado a cabo cuando se ha hablado de la finalidad, y, aunque está claro que una gramática descriptiva de una lengua histórica no debe tener por objeto la definición universal de conceptos, sí necesita de ellos como instrumental previo para la

2 A esta cuestión habría que añadir un inconveniente más con el que cuentan la mayoría de los trabajos lingüísticos: la ausencia de límites precisos entre las nociones de causa y finalidad, cuyo origen se remonta a la antigüedad clásica. Es precisamente Aristóteles en sus escritos el que, al tratar el problema de la causa, de su naturaleza y de sus especies (aunque es éste un aspecto al que alude muy a menudo en su obra, destacan principalmente sus formulaciones en Aristóteles, 1973: 65 y 1994: 79-80, 207-211), distingue entre los siguientes tipos de causas: 1) la causa formal (causa formalis), que es la entidad, esto es, la esencia, idea o cualidad de la cosa en cuestión; 2) la causa material (causa materialis), es decir, el sujeto o aquello de lo cual algo surge o mediante lo cual llega a ser; 3) la causa eficiente (causa efficiens), que es aquélla de donde proviene el movimiento (el principio del cambio), y 4) la causa final (causa finalis), opuesta a la anterior y que expresa aquello para lo cual (la realidad hacia la cual algo tiende a ser). Esta doctrina aristotélica sobre la naturaleza de la causa y las especies de ésta se conserva en el pensamiento escolástico y tiene su posterior repercusión en los estudios lingüísticos, en los que se producen continuas interferencias entre las nociones de finalidad y causa, sin que se aborde el estudio de cada una de ellas como nociones independientes que den cuenta de la existencia de dos fenómenos lingüísticos diferenciados. 
correcta identificación de la extensión ${ }^{3}$ de éstos y la descripción de las características de los objetos que entran a formar parte de los mismos en una lengua determinada. ${ }^{4}$

A pesar de que con el término 'finalidad' no se alude en el ámbito de la lingüística, como venimos comentando, a un concepto instrumental del que podamos valernos correctamente para su uso en la teoría lingüística, ello no implica la inutilidad del término para la explicación lingüística, ya que si -como defiende E. Coseriu (1967: 238)- el lenguaje es usado primordialmente como logos semánticos, es decir, como medio de expresión con intención comunicativa, habrá que admitir la existencia de una función comunicativa en las lenguas que podemos denominar finalidad, pues no se infiere la misma intención comunicativa en expresiones como Me gusta hacer la compra por las mañanas o Ayer me marché de tu casa a las ocho que en Me gusta hacer la compra por las mañanas para tener libre la tarde o Ayer me marché de tu casa a las ocho para no llegar tarde al trabajo, donde por medio de la introducción de los constituyentes finales el enunciador aporta una nueva dimensión expresiva en su enunciado que altera, obviamente, la finalidad comunicativa del mismo.

El problema en este punto está, así pues, en saber cómo delimitar la finalidad como concepto lingüístico, pues aunque podamos afirmar su existencia como tal, hasta el momento no parece que en el terreno de la lingüística hayamos sabido caracterizarlo. En las líneas que siguen no insistiremos más en los problemas que presentan las distintas perspectivas que hasta la actualidad se han barajado en la interpretación de la finalidad (cf., al respecto, V. Gaviño Rodríguez, 2009a). Nuestro estudio se centrará en lograr la definición de la finalidad como concepto lingüístico universal mediante su delimitación como noción que forme parte de una teoría del lenguaje, esto es, como instrumento válido para la posterior descripción de los objetos particulares que en las lenguas particulares (en nuestro caso en español) la representan.

\section{La finalidad como concepto lingüístico. Teoría del conocimiento y conceptualización}

La labor de delimitación de la finalidad como término lingüístico pasa por atender, en primer lugar, al proceso de conceptualización, que representa un papel primordial y vital en la clasificación de objetos, al ser el único sistema por medio del cual éstos son encuadrados en nuestra experiencia anterior al mundo. Conceptualizar es desarrollar o construir ideas abstractas a partir de la experiencia pero también es un mecanismo de reconocimiento de lo percibido. Y es precisamente así como operamos todos nosotros cuando reconocemos como un niño, como un coche, como un árbol, etc., las distintas cosas que percibimos. Toda percepción humana incluye la conceptualización de lo percibido, la aplicación de conceptos que, poco a poco, configuran una amplia red de conceptos por medio de los cuales clasificamos la multiplicidad y variedad que percibimos diariamente. El concepto supone, así pues, situarse en una perspectiva abstracta, en una unidad de pensamiento que se genera por medio de la agrupación de objetos individuales relacionados entre sí por características comunes. Por esta cuestión ya se interesó E. Husserl, cuya respuesta a cómo se constituyen en la percepción tales asignaciones desde la percepción de objetos particulares la encontramos en sus propias palabras:

\footnotetext{
3 La suma de las características que definen un concepto y lo distingue de los demás conceptos de la estructura conceptual a la que pertenece constituye su intensión, mientras que denominamos extensión a los objetos del mundo extralingüístico a los que el concepto hace referencia.

4 Para una visión pormenorizada de los problemas que conlleva la mayoría de estos estudios lingüísticos, cf. algunos trabajos anteriores en Gaviño Rodríguez (2001), (2002), (2003), (2004) y (2007a).
} 
Nosotros, desde nuestro punto de vista, nos colocaríamos, por de pronto, en una esfera de la abstracción sensible - preferida hasta ahora por su sencillez-, y en ella llevaríamos a cabo una distinción entre los actos en los cuales está "dado" intuitivamente un momento atributivo y los actos construídos (sic) sobre éstos. Estos segundos actos no son actos de mera atención a dicho momento, sino actos nuevos que, generalizando, mientan la especie correspondiente (E. Husserl, 1929: 167).

La función del concepto consiste, así pues, en flanquear los límites que nos impone la inabarcable multitud de las singularidades individuales. Por medio de los conceptos, tenemos la posibilidad de considerar las cosas en haces - por utilizar la terminología del propio E. Husserl (1929: 172) - y hacer referencia así a clases enteras, esto es, a innumerables objetos, en lugar de aprehender y juzgar cada objeto por sí. Los conceptos serían representaciones mentales que nos sirven para clasificar los numerosos objetos individuales. Se hace necesario, en este punto, establecer una diferenciación entre el concepto propiamente dicho, los objetos de la realidad que representan tales conceptos y el término que sirve de base a la comunidad de especialistas para designar los objetos de esa realidad ya existente. Resulta evidente que la esencia de un perro, un árbol, lo cómico, un círculo, una elipse, etc., se realiza en seres o situaciones concretas, pero, subsista o no fuera de esas situaciones concretas, se la puede describir como si fuera independiente de ellas. Es, si se nos permite decirlo así, una cierta naturaleza posible que no puede nacer poco a poco, sino que se nos presenta de golpe. La operación de conceptualización, que es indispensable para reconocer como tales un perro, un árbol, lo cómico, un adulto, un cuadrado, un triángulo, un círculo o una elipse, no impide que sea también posible formar o construir nuevos conceptos a partir de la experiencia posterior con operaciones mentales como, por ejemplo, caer en la cuenta de ciertas relaciones de semejanza entre objetos (relaciones anteriormente no advertidas), que puedan modificar, precisar o enriquecer nuestro concepto inicial. La adquisición de nuevos conceptos es algo fundamental para la ampliación de nuestros conocimientos y los progresos científicos, por su parte, siempre van acompañados de la creación de conceptos nuevos.

El proceso de conceptualización, viejo conocido en las teorías filosóficas del conocimiento, es escasamente aplicado en el ámbito de la lingüística, a pesar de ser quizás la fuente primordial para las definiciones de los conceptos representados por la terminología usada en el desarrollo de la función metalingüística ('finalidad', 'causalidad', 'semántica', 'oración', 'paradigma', 'sintagma', etc.). Estas unidades constituyen el delicado terreno de la terminología lingüística, usada por la mayoría de los estudios lingüísticos sin que el proceso de conceptualización al que anteriormente hemos aludido sea generalmente tenido en cuenta. De este modo, términos como 'finalidad' (pero también muchos otros que aquí no tratamos por razones evidentes, caso de 'complemento indirecto', 'sujeto', 'causa', 'afectado', etc.) nunca han sido considerados como designadores de verdaderos conceptos. Para llevar a cabo la definición de tales conceptos es necesario seleccionar las características que son comunes a una pluralidad de objetos y prescindir de aquellas características que no comparten todos ellos. Para llegar a las caracterizaciones de causa, finalidad, oración, etc., como términos lingüísticos, no parece que exista otro camino que el de atender, más que a los múltiples e inabarcables objetos reales que se representan bajo tales denominaciones, a sus respectivos conceptos. Así, del mismo modo que en la geometría el concepto de triángulo recoge las características que son comunes a todos los triángulos (aquellas características sin las cuales

\footnotetext{
Evidentemente, sólo un número escasísimo de lo que podría ser observado se observa realmente con el interés del investigador -como apunta K. Bühler $\left({ }^{3} 1967\right.$ : 52)- peculiaridad que no debemos pensar que es exclusiva de la lingüística. Todas las ciencias que parten de la experiencia son iguales entre sí en que, partiendo para su objeto de estudio de un inabarcable banco de objetos determinables, eligen sólo muestras apropiadas para llegar, a partir de ellas, a la determinación y el análisis científico. A partir de ese hecho, se trabaja con la suposición de que con ese poco se puede abarcar la totalidad.
} 
un objeto dejaría de serlo), dejando fuera aquellas otras que no son comunes a todos ellos (hay triángulos grandes y pequeños, los hay de mayor o menor área) por tratarse de características no pertinentes, el concepto de finalidad en el ámbito lingüístico ha de reunir sólo y exclusivamente aquellas características comunes a todos los casos que se representan bajo tal denominación, sin excluir ninguno de ellos. La tendencia general de los estudios lingüísticos es, contrariamente, concentrarse en la enumeración de rasgos concretos para los fenómenos en cada lengua concreta, sin deslindarse en ellos lo pertinente de lo anecdótico. La consecuencia de este modo de proceder, en el que continuamente tropezamos con incoherencias y contradicciones, es la ausencia de una delimitación de los conceptos abordados.

\subsection{Lo universal y lo necesario}

Aristóteles asigna dos características formales al saber para que éste pueda presentarse como científico. Estas dos características específicas del conocer científico (no queremos con ello, lógicamente, ser completamente exhaustivos, pero sí enderezar lo máximo posible nuestras reflexiones y acercamiento al estudio lingüístico) son que este ha de ser un conocimiento de lo necesario y estar basado en lo universal, pues no puede haber ciencia de lo contingente (cf. Aristóteles, 1994: 270-274). Si lo que pretendemos es que el conocimiento científico sea absolutamente válido, está claro que deberá expresar en sus juicios la necesidad de que su objeto es de una determinada forma y no de otra. ${ }^{6}$ Por ello, la correcta caracterización del concepto de finalidad no tiene otro camino que el de su delimitación por medio de aquellas características que lo son de manera absoluta, o sea, de las que son así y no de otro modo, por medio de su necesidad (lo necesario para ser tal objeto). Lo necesario ha de producirse efectivamente en todos y cada uno de los casos, siendo por tanto lo común que los vincula. Puesto que la ciencia trata de subsumir la multiplicidad ofrecida a los sentidos en unos cuantos parámetros que expliquen con veracidad y relativa sencillez el panorama de la multiplicidad, hallando lo común a todo ello y que por lo tanto existe y se da permanentemente, es lógico que se tenga eso también en cuenta para un estudio de la finalidad. Es evidente que la sensación, vinculada necesariamente a lo particular, no puede proporcionar ciencia. Así, lo universal es lo que siempre se da en todas partes y lo que no es universal no es tampoco necesario y por ello, no puede proporcionarnos un conocimiento científico, estable y permanente, sino uno circunstancial y contingente. El propio Aristóteles especifica sin lugar a dudas esta vinculación entre lo universal y lo necesario, al mismo tiempo que aclara su concepción de lo universal: entiende por universal lo que está en todos, en cada uno por sí, y en cuanto tal (cf. Aristóteles, ${ }^{3} 1978$ : 120). Así pues, tres son las características de lo universal: en primer lugar, lo que se da en todos, lo absolutamente común y que no admite excepción, por lo cual es permanente y estable, y responde claramente como posible objeto de ciencia. En segundo lugar, eso que está en todos está en cada uno de ellos no de manera accidental, sino respondiendo esencialmente a lo que es así, como requisito

6 Aristóteles distingue diversos tipos de necesidad: aunque en el Libro XII de la Metafísica (cf. Aristóteles, 1994: 487) distingue de manera general tres acepciones de 'necesario' (lo que se produce violentamente, al ser contrario a la inclinación, aquello sin lo cual no se produce el bien y lo que no puede ser de otro modo, sino que absolutamente es como es), previamente en el Libro V (cf. Aristóteles, 1994: 215-217) señala cinco: 1) aquello sin lo cual no se puede vivir (por ejemplo, la respiración y la alimentación); 2) aquellas cosas sin las cuales el bien no puede existir o producirse, o el mal no puede desaparecer (por ejemplo, el beberse la medicina es necesario para no estar enfermo); 3) lo impuesto con violencia en contra de la tendencia definida del ser (un movimiento violento forzado, por ejemplo); 4) lo necesario como lo que no puede ser de otra forma a como es, y 5) la demostración de las cosas necesarias en tanto que se ha demostrado estrictamente. Es la acepción cuarta la que alude a la forma de necesidad a la que se refiere la ciencia: la que no puede ser de otro modo, sino que es absolutamente (á $\pi \hat{\omega} \tilde{\omega} \varsigma$ simpliciter). 
fundamental de su ser, por lo tanto, de manera necesaria. En tercer lugar, y como confirmación de lo anterior, está en cada uno en cuanto tal, o sea, en cuanto es eso y no otra cosa, por tanto de manera esencial y necesaria. Lo universal, de esta forma, es algo común que se presenta siempre de manera necesaria, y por eso es casi una derivación consecuente de lo que es necesario. Si para la delimitación de la finalidad no tenemos en cuenta estas observaciones, cometeríamos el error de no expresar o no incluir en ella características siempre válidas, fijas y permanentes, determinándose una noción desprovista de rigor y sujeta a las múltiples contradicciones y problemas con los que con frecuencia nos venimos encontrando en los estudios lingüísticos.

En esta misma línea también habría que situar los conceptos de universalidad y generalidad propuestos por E. Coseriu en relación con la gramática. Para E. Coseriu (1981: 54-56), toda gramática ha de ser, no general, sino universal (conceptual o esencial) en cuanto teoría de conceptos gramaticales y en cuanto modelo de gramática válido para cualquier lengua histórica, sin que ello implique su generalidad empírica: "lo general es el conjunto de caracteres constantes que, teniendo ya el concepto, comprobamos efectivamente en una clase de objetos; caracteres que pueden también no ser indispensables para que los objetos sean lo que son: simplemente se dan en ellos", mientras que la universalidad es entendida como "el modo necesario de ser de tales y cuales objetos: lo que pertenece al concepto de un objeto o puede deducirse del concepto, o sea, el conjunto de aquellos caracteres sin los cuales un objeto no sería lo que es" (E. Coseriu, 1981: 54). El corolario de su planteamiento es que este modo de ser no se forma por abstracción, ya que esa operación implicaría una intuición previa de lo universal, sino por intuición inmediata de un modo de ser.

En virtud de lo comentado, se deduce la necesidad de operar con conceptos, en tanto representaciones mentales de objetos, en virtud de un proceso de selección de las características relevantes que definen a cada clase de objetos. Estas no son otras que las marcadas por el principio de universalidad anteriormente formulado: el concepto queda integrado por el conjunto de propiedades que diferencian de manera necesaria y suficiente entre una determinada clase de objetos y otra. Ello no supone, no obstante, que la experiencia posterior pueda modificar, precisar o enriquecer nuestro concepto inicial, circunstancia ya señalada con anterioridad y que es admitida por el propio E. Coseriu. Ahora bien, la universalidad conceptual nos impide hablar de objetos pertenecientes a una lengua histórica. Cuando hablamos de conceptos nos referimos a posibilidades universales del lenguaje que pueden presentarse (lo que no significa que lo hagan) en cualquier lengua. Las definiciones pertenecen no al ámbito de lo material o físico, sino al de lo mental, a la interioridad de la conciencia, de ahí que la finalidad, como concepto gramatical, habrá de ser universal en el sentido establecido y sólo así podrá ser definida. Cuando hablamos de una lengua en particular, en nuestro caso del español, debemos decir si tal o cual categoría se presenta o no se presenta en la lengua y, si se presenta, entonces sí podremos precisar sus funciones y describir -nunca definir- su forma material. ${ }^{7}$

7 Debemos aceptar que las descripciones nunca son completas y sus explicaciones nunca son definitivas, porque siempre se nos escapa un número ilimitado de variables. Además en ellas surge, como ya se ha comentado, la inesperada emergencia de la novedad. Incluso la más cuidadosa descripción puede pasar por alto importantes novedades que no permanecerían inadvertidas en una descripción exacta: como suele decirse, es fácil darse cuenta de las cosas después que han ocurrido. Claro está que la incertidumbre que caracteriza la descripción podría eliminarse o disminuirse si se contara con un conocimiento más completo de las variables y datos pertinentes para el caso. Pero la realidad es que frecuentemente se llega a descripciones con la ayuda de un número fijo y hasta reducido de datos. Todo ello lleva a que algunos autores, caso de K. Popper (1974: 17) y (1998: 45), cuestionen la validez de los enunciados universales de las ciencias empíricas. 


\subsection{Definición de la finalidad como concepto lingüístico y su descripción en español}

En el VI Congreso Internacional de la Sociedad Española de Historiografía Lingüística, celebrado en Cádiz entre el 6 y el 9 de noviembre de 2007, ya criticábamos las continuas relaciones que a lo largo de la historia de la lingüística se vienen realizando entre las nociones de 'causa' y 'finalidad', cuyo origen hay que atribuir a la obra de Aristóteles y que, desde nuestro punto de vista, constituyen el origen de la falta de delimitación y distinción entre ambas nociones desde el punto de vista lingüístico (cf., al respecto, V. Gaviño Rodríguez, 2009b: 271-288). Ello no quiere decir que los planteamientos aristotélicos sean del todo desechables. Contrariamente, a pesar de no coincidir con su visión conjunta de causa y finalidad, nuestra definición de finalidad parte de su caracterización de la denominada por él causa final (aquella para lo cual, es decir, la realidad hacia la cual algo tiende a ser), opuesta a la causa eficiente (aquella de donde proviene el movimiento, esto es, el principio del cambio). Partiendo de esta diferencia, aunque sin coincidir con Aristóteles en el planteamiento conjunto de estas dos nociones, la finalidad es, como término lingüístico, lo que se concibe como aquello para lo cual sucede lo que, a su vez, se concibe como un modo de suceder. Frente a ella, la causa es lo que se concibe como aquello por lo cual sucede lo que, a su vez, se concibe como un modo de suceder. De este modo, ante dos enunciados como Cogió el paraguas porque llovía y Voy al parque para que me cuentes todo, podemos decir que, en el primer caso, porque llovía se concibe como aquello por lo que sucede lo que, a su vez, se concibe como un modo de suceder (porque llovía expresa la causa de la acción de coger el paraguas); en el segundo ejemplo, para que me cuentes todo se concibe como aquello para lo cual sucede lo que, a su vez, se concibe como un modo de suceder (para que me cuentes todo expresa la finalidad de la acción de ir al parque).

Mientras que en la expresión de la causa centramos nuestro interés en lo que, en terminología aristotélica, es el principio (o motor) que promueve el cambio, en la expresión de la finalidad nos centramos en el hecho al que se tiende. Esta dualidad se nos antoja sumamente útil si queremos explicar las diferencias significativas existentes en enunciados muy similares, como, por ejemplo, Sara cocina porque quiere impresionar a sus invitados y Sara cocina para impresionar a sus invitados. Según lo anterior, en el primer caso tenemos una expresión causal: porque quiere impresionar a sus invitados se concibe como aquello por lo que sucede lo que, a su vez, se concibe como un modo de suceder (la acción de 'cocinar') porque como enunciador centramos nuestro interés en el principio (o motor) que promueve el cambio, esto es, en el suceso de 'querer impresionar a sus invitados'; en el segundo enunciado, para impresionar a sus invitados no se expresa ya una causa, sino una finalidad, esto es, aquello para lo que se produce dicha acción ('impresionar') porque ahora el enunciador centra la atención en el suceso de 'impresionar' como el hecho al que se tiende, siendo la acción de 'cocinar' el origen que lo propicia.

Si nos centramos en la perspectiva que adopta el enunciador con respecto al mensaje que emite, vemos que este, al emitir el enunciado, sitúa la finalidad en una posición de posterioridad lógica con respecto al otro suceso expresado; la causa, sin embargo, es situada por el enunciador en una posición anterior con respecto al otro suceso expresado. En esta línea (continuamos con los ejemplos anteriores), en el primer enunciado tenemos la expresión de la causa porque el enunciador dice que Sara cocina y ese hecho ya tiene como suceso anterior la intención de querer invitar a sus invitados; en el segundo, con expresión de finalidad, el enunciador dice que Sara cocina y luego ese hecho tendrá como suceso posterior que sus invitados se impresionan, de ahí el valor final adjudicado.

Habrá que notar que una definición de la finalidad como la anterior presenta numerosas ventajas con respecto a otras que podamos encontrar en otros trabajos, fundamentalmente, porque con ella no hacemos referencia directa a la realidad extralingüística ni tampoco 
aludimos a aspectos descriptivos de una lengua concreta. El concepto de finalidad aportado no hace referencia a objetos particulares de las lenguas y puede ser aplicable a cualquier lengua histórica, ya que con él se establece una posibilidad universal del lenguaje que puede o no presentarse en cualquier lengua. Que definamos la finalidad como lo que se concibe como aquello para lo cual sucede lo que, a su vez, se concibe como un modo de suceder no implica que tengamos que vincular la finalidad exclusivamente con aquellas estructuras introducidas mediante la preposición para, como se ha hecho en algunos estudios gramaticales del español, puesto que una definición, como ya hemos advertido previamente, no equivale a la descripción de objetos concretos de una lengua concreta, sino que alude a un modo de ser universal de los objetos. Por lo tanto, con la definición anterior no se están describiendo objetos de la lengua española (aquellos introducidos por la preposición para), sino que se alude a un modo de ser universal de las lenguas. Lo que ocurre es que para designar ese modo de ser se ha empleado en la definición, circunstancialmente, la preposición para en español (del mismo modo que a lo mejor tendríamos que emplear otro nexo si para la emisión de nuestra definición usamos otra lengua), pero ello no significa ni que sólo existan finalidades en español ni tampoco que las unidades que representan en español la finalidad tengan que ir introducidas obligatoriamente por dicho nexo. Éstas pueden ir introducidas en español de manera muy diferente y, aunque de entre todos esos introductores son quizás para y $a$ los de mayor aparición y aceptación en este tipo de estructuras, no debemos olvidar la posibilidad de que la finalidad pueda ser expresada por medio de segmentos introducidos por nexos como, por ejemplo, por (que), en (que), de (que), que o locuciones del tipo a fin de (que), con el fin de (que), con el objeto de (que), en aras de (que), a por, etc.

Conviene por ello diferenciar claramente el modo de ser que denominamos 'finalidad' (universal y no intralingüístico) de los objetos que en las lenguas particulares puedan expresar dicho modo de ser, cuya identificación, ya desde un punto de vista descriptivo, podrá llevarse a cabo mediante el uso de determinadas pruebas. En el caso del español, podemos decir que, desde el punto de vista descriptivo, toda finalidad equivale al interrogativo ¿Con qué finalidad sucede algo? y que la expresión en la que ésta aparece responde a la pregunta de ¿Qué sucede con determinada finalidad? Así, por ejemplo, ante una expresión como Luisa se prepara para competir podríamos preguntar ¿Con qué finalidad se prepara Luisa?, a lo que responderíamos Con la de competir y, del mismo modo, a la pregunta de ¿Qué sucede con la finalidad de competir? responderíamos Que Luisa se prepara.

Estas pruebas de identificación de la finalidad en una lengua concreta no se insertarían ya en la noción de finalidad, sino en una generalización de la finalidad en español que evita lo particular (como pueden ser los hechos de que varíe la preposición introductora, que el nexo introductor no sea una preposición o que ni siquiera exista tal nexo). En este sentido, también en Pedro se empecinó en ganar la carrera, La niña rabia por que le concedan el primer premio, Corre que te vea tu abuela guapa, Escóndete, no te vaya a ver aquí y Yo me abstuve de votar los constituyentes en ganar la carrera, por que le premien, que te vea tu abuela guapa, no te vaya a ver aquí y de votar se interpretan como constituyentes finales, es decir, todos ellos se ajustan al concepto ya delimitado (aquello para lo cual sucede que Pedro se empecinó, que la niña rabia, que ella corre, que él se esconde y que yo me abstuve, respectivamente) y responden, igualmente, a las pruebas de identificación presentadas para su inclusión entre los complementos finales:

Pedro se empecinó en ganar la carrera: ¿Con qué finalidad se empecinó Pedro? Con la de ganar la carrera ¿Qué sucede con la finalidad de ganar la carrera? Que Pedro se empecinó

La niña rabia por que le concedan el primer premio: $¿$ ¿Con qué finalidad rabia la niña? Con la de que le concedan el primer premio ¿Qué sucede con la finalidad de que le concedan el primer premio? Que la niña rabia 
Corre que te vea tu abuela guapa:

¿Con que finalidad corre? Con la de que la vea su abuela guapa

¿Qué sucede con la finalidad de que la vea su abuela? Que (ella) corre

Escóndete, no te vaya a ver aquí:

$¿$ ¿Con que finalidad se esconde? Con la de que (alguien) no la vaya a ver aquí

¿Qué sucede con la finalidad de que no la vayan a ver aquí? Que se esconde

Yo me abstuve de votar:

¿Con qué finalidad me abstuve? Con la de no votar

¿Qué sucede con la finalidad de no votar? Que yo me abstuve ${ }^{8}$

Contrariamente, en otros enunciados formalmente similares no podemos hablar de la existencia de constituyentes finales. Es lo que ocurre en las expresiones Estoy muy cansado para hablar ahora de eso, Cierra la ventana que tengo frío, Empieza por hacer una lista de responsabilidades, El tren está para salir o Jaime se abalanzó a besar a su hermana y salió disparado, donde no identificamos segmentos con valor de finalidad porque ninguna de ellas cumple con la definición de finalidad que aportamos en este estudio: ni los segmentos para hablar ahora de eso, que tengo frío, por hacer una lista de responsabilidades, para salir o a besar a su hermana pueden ser concebidos como aquello para lo cual sucede que estoy muy cansado, que cierra la venta, que empieza, que el tren está o que Jaime se abalanzó, ni tampoco en tales enunciados se cumplen las pruebas descriptivas de identificación de los complementos finales en nuestra lengua:

Estoy muy cansado para hablar ahora de eso:

¿Con qué finalidad estoy muy cansado? *Con la de hablar ahora de eso

¿Qué sucede con la finalidad de hablar ahora de eso? *Que estoy muy cansado

Cierra la ventana que tengo frío:

¿Con qué finalidad cierra la ventana? *Con la de que tengo frío

¿Qué sucede con la finalidad de que tengo frío? *Que cierra la ventana ${ }^{9}$

Empieza por hacer una lista de responsabilidades:

¿Con qué finalidad empieza? *Con la de hacer una lista de responsabilidades

¿Qué sucede con la finalidad de hacer una lista de responsabilidades? *Que empieza

8 Enunciados como el propuesto Yo me abstuve de votar, que tradicionalmente han carecido de un análisis correcto en muchos estudios, son también ejemplos de expresiones con constituyentes finales, si bien los verbos a los que éstas determinan poseen una peculiaridad semántica que hace diferente esta combinatoria entre verbo y complemento final. Verbos como abstenerse, desistir, dispensar o disuadir poseen una carga semántica negativa que obliga a la inclusión de la negación en la aplicación de las pruebas de identificación de la finalidad en nuestra lengua. En este sentido, si ante el enunciado Yo me abstuve de votar preguntamos ¿Con qué finalidad me abstuve?, no podemos responder Con la de votar, pues estaríamos así invirtiendo el sentido de la expresión originaria. Precisamente por esa peculiaridad semántica, la respuesta correcta a este tipo de enunciados sería Con la de no votar. Del mismo modo, en la pregunta a la expresión en la que se incluye la finalidad incluimos nuevamente la negación, como vemos en ¿Qué sucede con la finalidad de no votar?, pregunta a la que respondemos con que me abstuve. Idéntica situación tenemos para el resto de expresiones textuales con otros verbos similares, caso de los ya mencionados desistir, dispensar y disuadir.

9 Aunque enunciados del tipo de Cierra la ventana que tengo frío y Corre que te vea tu abuela guapa, muy frecuentes en el habla coloquial, puedan parecer formalmente idénticos, lo cierto es que, conceptualmente, la interpretación de los constituyentes que tengo frío y que te vea tu abuela guapa en cada uno de sus enunciados difiere plenamente: que te vea tu abuela guapa es concebido como aquello para lo cual sucede la acción de correr y además en dicho enunciado se cumplen las pruebas de identificación de finalidad $(¿$ Con que finalidad corre? Con la de que la vea su abuela guapa; ¿Qué sucede con la finalidad de que la vea su abuela? Que (ella) corre); en el caso de que tengo frío, este segmento no puede ser concebido como aquello para lo cual sucede la acción de cerrar la ventana, y tampoco en el enunciado se cumplen las pruebas identificadoras de finalidad (¿Con qué finalidad cierra la ventana? *Con la de que tengo frío; ¿Qué sucede con la finalidad de que tengo frío? *Que cierra la ventana). Contrariamente, estamos ante un enunciado con complemento de causa: que tengo frío se concibe como aquello por lo cual sucede que alguien cierra la ventana. 
El tren está para salir:

¿Con qué finalidad está el tren? *Con la de salir

¿Qué sucede con la finalidad de salir? *Que el tren está

Jaime se abalanzó a besar a su hermana y salió disparado:

¿Con qué finalidad se abalanzó Jaime? *Con la de besar a su hermana

¿Qué sucede con la finalidad de besar a su hermana? *Que Jaime se abalanzó ${ }^{10}$

Junto a la definición de finalidad, la presencia en la teoría de unas pruebas para la identificación y descripción de estos complementos finales en cada una de las lenguas (nosotros las hemos propuesto para el español) hace posible que podamos interpretar como claramente finales diversos segmentos que en muchos casos han sido centro de la polémica en el estudio de la finalidad. Entre otros casos, podemos hacer referencia a aquellos que se combinan con estructuras en las que no contamos con la voluntad o intencionalidad de un sujeto caracterizado por el rasgo 'humano' o los que acompañan a estructuras oracionales a las que subyace un esquema no activo, entre otros casos. Pasamos a analizar algunas de estas situaciones.

El origen de la necesidad de un sujeto marcado por el rasgo 'humano' portador de la intencionalidad o voluntad para la presencia de constituyentes finales en español es una característica que conservamos como legado de la filosofía en relación con la clásica distinción entre el finis operis y el finis operantes, cuya presencia exige, desde el punto de vista filosófico, la de un ser dotado de algún tipo de consciencia y, en sentido estricto, la del hombre, ya que es el único con la capacidad de reflexionar sobre sus tendencias y planificar, de manera intencionada, la finalidad de sus acciones.

Desde esta perspectiva, en el ámbito de la lingüística hispánica, se ha defendido -cf., por ejemplo, la visión de algunos autores como A. Narbona (1990: 54-55), M. Fernández Lagunilla y A. Anula Rebollo (1995: 340), C. Hernández Alonso (1995: 155), S. García (1996: 63-64) o C. Galán (1999: 3621)- que, puesto que la finalidad posee un carácter intencional y es entendida como un proceso que ha de contar con la voluntad (que sólo es atribuible a las personas), su aparición en un enunciado está condicionada a la presencia (explícita o implícita) de un agente caracterizado por el rasgo 'humano'. ${ }^{11}$

Desde nuestro punto de vista, la presencia de constituyentes finales en estructuras en las que exista intencionalidad por parte del ser designado por el tradicionalmente denominado sujeto no es más que una característica que hace referencia al mundo extralingüístico, pero en modo alguno parece que sea una necesidad basada en exigencias de tipo lingüístico. Aunque son muchos los enunciados en que parece que la lengua interpreta esa realidad y nos la muestra como si fuera un espejo de la misma, no es menos cierto que la asimetría está presente en otros muchos enunciados (por ejemplo, Las señales están ahí para dirigir el tráfico o La manta está encima de mi cama para que yo no pase frío por las noches) sin que con ello se niegue, en modo alguno, la presencia de finalidad en la lengua: las funciones expresadas por los segmentos para dirigir el tráfico y para que yo no pase frío por las noches pueden ser las finalidades tanto de Las señales están ahí como de La manta está encima de mi cama, por más que los seres designados por las señales o la manta carezcan de intencionalidad

10 En los tres últimos ejemplos, Empieza por hacer una lista de responsabilidades, El tren está para salir y Jaime se abalanzó a besar a su hermana y salió disparado nos encontramos con la presencia de estructuras perifrásticas de verbo + preposición + verbo en las que el verbo principal de cada estructura (empezar, estar, abalanzarse) ha pasado del nivel de las unidades léxicas al de las gramaticales -así lo afirma W. Dietrich (1973: 578) - llegando a ser distintivo desde la perspectiva instrumental o gramatical. Para un estudio más detallado de estas falsas construcciones finales en español, cf. V. Gaviño Rodríguez (2007b) y (2008).

11 S. García (1996: 63-64), por su parte, posee una visión algo más amplia al defender que la intencionalidad es una característica atribuible a las personas y animales, pero no a objetos ni a entes conceptuales. 
alguna en la realidad a la que aluden. ${ }^{12}$ Aunque las expresiones finales se asocian frecuentemente con la presencia de sujetos animados, también es posible su aparición en otros esquemas como los subyacentes a enunciados como Este botón sirve para encender el ordenador, Estos ojos son tan grandes para verte mejor, etc., como ha manifestado también recientemente J. Cuartero Otal (2003: 126, n. 114). Este tipo de finalidades son presentadas lingüísticamente como simples sucesos accidentales o casuales, y responden a la interpretación de fenómenos dirigidos a un fin que podemos denominar funcional (como el hecho de que el corazón está para que circule la sangre, el que las señales estén en algún lugar para dirigir el tráfico y evitar así accidentes, que la manta esté encima de mi cama para que yo no pase frío por las noches, etc.), por más que frecuentemente tras los sucesos que designan estos enunciados encontremos las actuaciones de determinados agentes que obran por un fin, ya que en tal caso estaríamos haciendo un análisis de la realidad, no de la lengua.

En enunciados sin sujetos animados, del tipo de Las señales están ahí para dirigir el tráfico o La manta está encima de mi cama para que yo no pase frío por las noches, aunque ni las señales ni las mantas tienen intenciones en la realidad ontológica, los hechos de que las señales o las mantas estén en algún lugar son concebidos, lingüísticamente, como sucesos dirigidos a un fin concreto, dirigir el tráfico y que yo no pase frío por las noches, respectivamente. Otro ejemplo: imaginemos que un terremoto produce una corriente de tierras y de rocas, lo que a su vez produce la desviación del curso de un río y esto permite luego edificar allí una ciudad. Si para aludir a ese hecho tenemos un enunciado como El terremoto se produjo para que pudiéramos construir nuestra ciudad, no tiene sentido pensar que el terremoto actuara con el fin de desviar el curso del río, y mucho menos, que el suceso de que se produjera tal terremoto fuera el medio para edificar allí una ciudad. Ahora bien, ello no obsta para que en un enunciado como El terremoto se produjo para que pudiéramos construir nuestra ciudad podamos sostener que, lingüísticamente, el segmento para que pudiéramos construir nuestra ciudad es concebido como aquello para lo cual sucede lo que a su vez concebimos como el suceso de que el terremoto se produjera. El enunciador, al emitir tal enunciado, sitúa el suceso de que el terremoto se produjo en una posición de anterioridad lógica con respecto al suceso expresado por para que pudiéramos construir nuestra ciudad.

Que la noción de intencionalidad no tiene cabida en la de finalidad lo demuestra además la existencia de complementos finales con sujetos marcados con el rasgo 'humano' que pueden designar el resultado ciego y hasta casual de un suceso (que se da de manera inconsciente o instintiva), sin que exista razón alguna para pensar que sea algo perseguido: si alguien sale de paseo y se encuentra una moneda, generalmente tal hallazgo no es un fin intentado, sino un simple resultado ocasional. Sin embargo, nadie tendrá dudas de que en el análisis de una expresión como Salí de paseo para encontrarme una moneda contamos con la presencia de un complemento final (para encontrarme una moneda), más allá de si la moneda fue o no buscada intencionalmente, cuestión que, lingüísticamente, carece de interés. Aunque en el ámbito de la realidad no lingüística, la persona a la que se designa en este enunciado (el yo) no haya salido a pasear con la intención de encontrar una moneda, desde una perspectiva lingüística, el hecho de que yo salga de paseo es concebido como un suceso que está dirigido a un fin concreto: encontrarme una moneda. En tal caso, el suceso con valor final se sitúa en una posición de posterioridad lógica con respecto al suceso expresado por salí de paseo: el enunciador nos dice que él salió de paseo y luego ese hecho tuvo como suceso posterior que se encontró una moneda. La intencionalidad de querer o no encontrar la moneda respondería a

12 Curiosamente, son algunos de estos mismos autores los que acaban aceptando la existencia de expresiones finales en este tipo de enunciados (por ejemplo, El sol sale todas las mañanas para alumbrarnos o La Unión Europea no consigue ponerse de acuerdo para ayudar a los agricultores), aunque para ello suelen intentar acomodar los ejemplos a la teoría, considerando la presencia de personificaciones o usos metafóricos en estos casos. 
un hecho de la realidad, pero no a la interpretación lingüística del enunciado propuesto, del mismo modo que, en un enunciado como Finalmente, bebió un litro de agua, no nos cuestionamos si la persona que bebió un litro de agua lo hizo intencionalmente porque tenía mucha sed, o sin ella porque le abrimos la boca y le obligamos a beber el litro como castigo por haber perdido una apuesta.

En relación con el anterior supuesto está el hecho de que aceptemos o no la existencia de expresiones finales en estructuras oracionales a las que subyace un valor no activo. En opinión de V. Báez San José (2002: 144, n. 104), en este tipo de enunciados (concretamente él ejemplifica con Los cojines son verdes para que hagan juego con el pavimento de la habitación) no tenemos constituyentes finales por el hecho de que son aceptables diálogos del tipo ¿Por qué son verdes los cojines? Para que hagan juego con el pavimento mientras que no lo son * ¿Para qué son verdes los cojines? Para que hagan juego con el pavimento.

$\mathrm{Su}$ argumento no nos parece, sin embargo, que esté sustentado en aspectos lingüísticos y nuestra opinión al respecto difiere de la suya y coincide en parte con la crítica formulada por P. P. Devís Márquez (2005: 364-365, n. 9) a esta perspectiva: que una expresión como Los cojines son verdes para que hagan juego con el pavimento de la habitación posee un constituyente final lo demuestra la propia existencia de otros tantos complementos finales en expresiones a las que subyace un valor no activo (por ejemplo, La bomba fue destruida para salvar a la humanidad o La manta está encima de mi cama para que yo no pase frío por las noches) y en las que se cumplen las pruebas identificadoras de finalidad en español. Por otro lado, tal y como también comenta P. P. Devís Márquez, a) no encontramos razones para no sostener que el enunciado Los cojines son verdes para que hagan juego con el pavimento de la habitación pueda ser una respuesta válida a la pregunta ¿para qué son verdes los cojines?, y b) en el caso de que fuera respuesta exclusiva a ¿Por qué son verdes los cojines?, como piensa Báez San José, ello tampoco implicaría la ausencia de finalidad, que, en función de lo ya comentado en este trabajo, no depende del nexo introductor para que; el valor de finalidad se conservaría incluso si cambiamos el nexo introductor de la expresión origen por por que. Tanto en Los cojines son verdes para que hagan juego con el pavimento de la habitación como en Los cojines son verdes por que hagan juego con el pavimento de la habitación hallamos un valor final, ya que, en ambos casos, para que hagan juego con el pavimento de la habitación y por que hagan juego con el pavimento de la habitación aluden a lo que se concibe como aquello para lo cual se produce lo que, a su vez, se concibe como un modo de suceder: la caracterización indicada por el verbo ser. En ambos casos, el enunciador sitúa el suceso de que hagan juego con el pavimento de la habitación en una relación de posterioridad lógica con respecto al suceso de que los cojines son verdes. Por otro lado, tanto en una como en otra expresión se cumplen las pruebas de identificación de la finalidad en nuestra lengua: ¿Con qué finalidad son verdes los cojines? Con la de que hagan juego con el pavimento de la habitación, y ¿Qué sucede con la finalidad de que hagan juego con el pavimento de la habitación? Que los cojines son verdes. ${ }^{13}$

13 En muchos casos encontramos dobletes de enunciados a los que subyace una estructura activa y no activa, respectivamente, como sucede en Mi mujer se prepara para competir profesionalmente y Mi mujer está siendo preparada por una máquina para competir profesionalmente, y donde se cumplen claramente las premisas de definición y descripción a la que venimos aludiendo en este trabajo: a) para competir profesionalmente cumple con la definición de la finalidad aportada, pues dicho segmento puede ser concebido como aquello para lo cual sucede tanto el que mi mujer se prepara como el que mi mujer está siendo preparada por una máquina, y b) en ambos casos se cumplen las pruebas de identificación aportadas para el español: para competir profesionalmente es la respuesta a las preguntas ¿Con qué finalidad se prepara mi mujer? y ¿Con qué finalidad está siendo preparada mi mujer?, y las expresiones en las que se incluye dicho complemento responden, igualmente, a ¿Qué sucede con la finalidad de competir profesionalmente? 
Independientemente de los nexos que las introduzcan o de la estructura formal de sus constituyentes, ${ }^{14}$ interpretamos como finales todas aquellas estructuras que se encuentren bajo el marco de nuestra definición y respondan a las pruebas de identificación presentadas. No hay que olvidar que la oposición básica entre la finalidad y la causa es que la finalidad se sitúa conceptualmente en una relación lógica de posterioridad con respecto al suceso al que ésta determina, es decir, se trata de algo posterior a dicho suceso. ${ }^{15}$ Con ello, sin embargo, no cancelamos los problemas interpretativos que encontramos en ciertas secuencias textuales. A pesar de que en el nivel oracional no hay lugar para ambigüedades, en el análisis del nivel textual pueden operar factores contextuales que dificulten la interpretación de determinados constituyentes. Por ejemplo, en Come porque quiere engordar un poco o Come para engordar un poco resulta fácil manifestarse a favor de que porque quiere engordar un poco expresa la causa, es decir, lo que se concibe como aquello por lo cual sucede lo que, a su vez, se concibe como la acción de comer (su fundamento o razón), mientras que para engordar un poco indica claramente la finalidad, esto es, aquello para lo cual sucede lo que, a su vez, se concibe como la acción de comer. No obstante, en un enunciado como Come por engordar un poco el constituyente por engordar un poco podría tener, en el discurso, una doble interpretación: 1) final, esto es, lo que se concibe como aquello para lo cual sucede lo que, a su vez, se concibe como la acción de comer (por engordar un poco posee un valor final porque el interlocutor, al emitir dicho enunciado, sitúa dicho suceso en una relación de posterioridad con respecto a la acción de 'comer', que le precede), o 2) causal, es decir, lo que se concibe como aquello por lo cual sucede lo que, a su vez, se concibe como la acción de comer (el valor causal de por engordar un poco viene determinado porque el interlocutor, al emitir dicho enunciado, sitúa el suceso al que alude en una relación de anterioridad con respecto a la acción de 'comer', que le sigue posteriormente). La posibilidad de esta segunda interpretación tiene su justificación en aspectos discursivos contextuales que incidan en la interpretación significativa de la expresión citada Come por (querer) engordar un poco. E idéntica situación se observa en otros enunciados como Duerme por fastidiarnos o No lo

14 Tampoco nuestra definición de finalidad tiene nada que ver con el número de finalidades que puede admitir cada verbo en un enunciado de una lengua concreta. Ése sería un problema, no de teoría lingüística, sino de gramática descriptiva de cada lengua, del mismo modo que lo es el hecho de que puedan existir lenguas en las que no se exprese finalidad, ya que la definición universal de un concepto gramatical no implica, como también se ha apuntado anteriormente, su generalidad. Que la definición aportada no obstaculiza en modo alguno el que en español un verbo pueda ir acompañado por más de una finalidad lo demuestra además la existencia de ejemplos como el último de los propuestos con dos finalidades (Lo indujo a matar para cobrar una herencia) si bien en muchos de estos casos cabe una doble interpretación y se hace necesario resolver la ambigüedad que puede producirse desde el punto de vista del sentido. En el caso concreto de Lo indujo a matar para cobrar una herencia, habría que defender, por un lado, que si el que induce es el mismo que va a cobrar la herencia, matar se concibe como aquello para lo cual sucede que alguien induce a alguien y cobrar una herencia se concibe como aquello para lo cual sucede que alguien induce a alguien a matar; por el otro, si el que va a cobrar la herencia no es el mismo que induce, sino el inducido, entonces matar para cobrar una herencia se concibe como aquello para lo cual sucede que alguien induce a alguien y cobrar una herencia sería lo que se concibe como aquello para lo cual alguien mata a alguien. Mientras que en el primer caso tenemos dos finalidades (a matar y para cobrar una herencia) referidas a un mismo modo de suceder (inducir), en el segundo caso la acción de inducir está determinada por una sola finalidad (a matar para cobrar una herencia) y la acción de matar se encuentra determinada, a su vez, por otra finalidad (para cobrar una herencia). Es precisamente esa doble interpretación textual la que permite que si preguntamos qué es aquello para lo que sucede la acción de inducir podamos responder, tanto Matar y Cobrar una herencia, como Matar para cobrar una herencia.

15 Precisamente porque la finalidad es concebida como algo posterior a un determinado suceso, siempre nos ha parecido ilógico hablar de una causa final. ¿Acaso no es contradictorio pensar que algo que todavía no existe pueda influir de modo causal? Otros autores no parecen plantearse este aspecto y defienden explícitamente una visión conjunta entre causa y finalidad. En esta línea, C. Galán (1999: 3621) sostiene que las expresiones finales manifiestan una relación de causa-efecto en la que el efecto se interpreta como posterior (propósito virtual), frente a la anterioridad (motivo realizado) que designa la causalidad. 
aceptó por ser un infeliz que, gracias a la indeterminación temporal de los infinitivos, pueden llevarnos a inferir valores causales o finales en los segmentos por fastidiaros o por ser un infeliz, según sean interpretados en el discurso como anteriores o posteriores lógicamente con respecto a los sucesos de 'dormir' y 'aceptar', respectivamente. Esta doble posibilidad interpretativa de los enunciados (aunque muchas veces viable) suele ser rápidamente resuelta en la comunicación por nuestro propio saber compartido o el conocimiento del mundo: así, en un enunciado como El equipo médico trabaja por su amistad, por ejemplo, no pensamos sistemáticamente en una doble posibilidad interpretativa para el segmento por su amistad. Nuestro conocimiento del mundo hace que ante un enunciado como el anterior rechacemos la posibilidad de considerar que el segmento por su amistad pueda ser considerado como la finalidad de la acción de trabajar (no responde, según nuestra experiencia, a aquello para lo cual sucede la acción de trabajar ni obedece a ninguna de las pruebas usadas para identificar los constituyentes finales en nuestra lengua: ¿Con qué finalidad trabaja el médico? ${ }^{*}$ Con la de su amistad; ¿Qué sucede con la finalidad de su amistad? *Que el médico trabaja) y eso hace que no nos parezca lógico pensar que la amistad sea aquello que persiguen los médicos con su trabajo, sino más bien la causa del mismo, su fundamento, razón o motivo (responde a aquello por lo cual sucede la acción de trabajar). Pero ello no quiere decir que, lingüísticamente, no exista la otra interpretación. Eso sí, para descubrirla tendremos que sobrepasar, una vez más, esa débil simetría entre mundo y lengua.

\section{Conclusión}

Con el presente trabajo hemos intentado delimitar la finalidad como noción lingüística, aportando además una serie de pruebas identificadoras de los constituyentes finales en español que nos sirvan para identificar correctamente estas frente a otras estructuras, fundamentalmente las causales. En un ámbito más general, hemos pretendido, igualmente, llamar la atención sobre el problema que la vaguedad terminológica o conceptual puede representar para el estudio de los fenómenos lingüísticos, haciendo hincapié en una serie de pautas siempre necesarias en la delimitación de un concepto.

Aquí no hemos trazado más que unas líneas que esperamos que sirvan para iniciar un camino hacia la definición y descripción de la finalidad, con el fin de lograr una herramienta útil que permita ir fijando la terminología que empleamos en nuestros trabajos y reducir, de esta manera, los problemas de ambigüedad o vaguedad conceptual que afectan con excesiva frecuencia nuestros trabajos. Somos conscientes de que algunas cuestiones han quedado en el tintero y que, a medida de que operemos con más variables, nuestras definiciones y descripciones se harán más precisas. ¿Pero qué mejor lugar que una conclusión para dejar entreabierta una puerta a nuevas ideas, nuevas investigaciones, en las que la incertidumbre de los datos hasta ahora registrados pueda verse disminuida con otros acercamientos que poco a poco arrojen luz para mejores interpretaciones de los fenómenos lingüísticos? 


\section{Bibliografía}

Aristóteles (1973): Physique, (I-IV) tome premier, texte établi et traduit par H. Carteron. Paris: Société d'Édition "Les Belles Lettres".

Aristóteles ( $\left.{ }^{3} 1978\right)$ : Analytica priora et posteriora, Recensuit brevique adnotatione critica instruxit W. D. Ross. Oxford: Oxford University Press.

Aristóteles (1994): Metafísica, introducción, traducción y notas de Tomás Calvo Martínez. Madrid: Gredos.

Báez San José, V. (2002): Desde el hablar a la lengua. Prolegómenos a una teoría de la sintaxis y la semántica textual y oracional. Málaga: Ágora.

Bühler, K. ( $\left.{ }^{3} 1967\right)$ : Teoría del lenguaje, traducción de J. Marías. Madrid: Revista de Occidente.

Coseriu, E. (1967): "Logicismo y antilogicismo en la gramática", en: Teoría del lenguaje y lingüística general. Madrid: Gredos, 235-260.

Coseriu, E. (1981): "El antipositivismo”, en: Lecciones de lingüística general. Madrid: Gredos, 52-73.

Cuartero Otal, J. (2003): Cosas que se hacen. Esquemas sintáctico-semánticos agentivos del español. Frankfurt am Main: Peter Lang.

Devís Márquez, P. P. (2005): "Algunos problemas en la teoría de los esquemas oracionales", en: Wotjak, G./ J. Cuartero Otal (eds.): Entre semántica léxica, teoría del léxico y sintaxis. Frankfurt am Main: Peter Lang, 357-372.

Dietrich, W. (1973): Der periphrastische Verbalaspekt in den romanischen Sprachen. Tübingen: Max Niemeyer.

Fernández Lagunilla, M. y A. Anula Rebollo (1995): Sintaxis y cognición. Introducción al conocimiento, el procesamiento y los déficits sintácticos. Madrid: Síntesis.

Galán Rodríguez, C. (1992): "Las oraciones finales en español. Estudio sincrónico", en: Anuario de Estudios Filológicos, Anejo nº 9. Cáceres.

Galán Rodríguez, C. (1993): Aproximación histórica al estudio de las oraciones finales en español. Cáceres: Servicio de Publicaciones de la Universidad de Extremadura.

Galán Rodríguez, C. (1999): "La subordinación causal y final", en: Bosque, I./ V. Demonte (dirs.): Gramática descriptiva de la lengua española. 3. Entre la oración y el discurso. Morfología. Madrid: Espasa Calpe, 3597-3642.

García, S. (1996): Las expresiones causales y finales. Madrid: Arco Libros.

Gaviño Rodríguez. V. (2001): "Sobre los denominados complementos circunstanciales finales", en: Veiga, A./Pérez, $\mathrm{M}^{\mathrm{a}} \mathrm{R}$. (eds.): Lengua española y estructuras gramaticales. Verba 48, Universidade de Santiago de Compostela, 171-181.

Gaviño Rodríguez, V. (2002): "Revisión historiográfica sobre la noción de finalidad en la tradición gramatical española”, en: Res Diachronicae 1, 178-189.

Gaviño Rodríguez, V. (2003): La finalidad en la tradición lingüística hispánica. Cádiz: Servicio de Publicaciones de la Universidad de Cádiz.

Gaviño Rodríguez, V. (2004): "La delimitación entre finalidad predicativa y finalidad extrapredicativa como problema del nivel textual", en: Villayandre Llamazares, M. (ed.): Actas del V Congreso de Lingüística General. Madrid: Arco Libros, 1301-1312.

Gaviño Rodríguez, V. (2007a): "Constituyentes obligatorios y constituyentes optativos: estudio de los complementos finales en español”, en: Zeitschrift für romanische Philologie 123, 2, 237-273.

Gaviño Rodríguez, V. (2007b): “A (que) y para (que) como introductores de complementos finales en español”, en: Bulletin of Hispanic Studies 84, 2, 113-130.

Gaviño Rodríguez, V. (2008): "Por (que) y para (que) como introductores de complementos finales en español”, en: Neuphilologische Mitteilungen (Bulletin of the Modern Language Society) 109, 2, 143-154.

Gaviño Rodríguez, V. (2009a): La finalidad como función lingüística. Oviedo: Septem Ediciones.

Gaviño Rodríguez, V. (2009b): "Causa, finalidad y causa final”, en: García Martín, J. Mª (dir.), Bastardín Candón, T. y M. Rivas Zancarrón (eds.): Estudios de Historiografía Lingüística. Cádiz: Servicio de Publicaciones de la Universidad de Cádiz, 271-288.

Hernández Alonso, C. (1995): Nueva sintaxis de la lengua española. Salamanca: Colegio de España.

Husserl, E. (1929): Investigaciones lógicas, II. Investigaciones para la fenomenología y teoría del conocimiento. Introducción e investigaciones I y II. Madrid: Revista de Occidente. 
Matte Bon, F. (1999): "Lingua, analisi della lingua e bi-logica", en: Bria, P./Oneroso F. (eds.): L'inconscio antinomico. Sviluppi e prospettive dell'opera di Matte Blanco. Milano: Franco Angeli, 88-132.

Matte Bon, F. (2007): "En busca de una gramática para comunicar", en: MarcoELE. Revista de didáctica, 5 .

http://www.marcoele.com/num/5/02e3c0996c1120f05/En_busca_de_una_gramatica_para_comuni car.pdf

Narbona Jiménez, A. (1985): "Finales y finalidad", en: Philologica Hispaniensia in honorem Manuel Alvar, II. Madrid: Gredos, 529-540.

Narbona Jiménez, A. (1990): Las subordinadas adverbiales impropias en español (II). Málaga: Ágora.

Popper, K. (1974): Conocimiento objetivo. Madrid: Tecnos.

Popper, K. (1998): Los dos problemas fundamentales de la epistemología. Basado en manuscritos de los años 1930-1933, edición de Troels Eggers Hansen y traducción de M ${ }^{\mathrm{a}}$ Asunción Albisu Aparicio. Madrid: Tecnos. 